\title{
Modelling knowledge in Electronic Study Books
}

\author{
C. Hoede \\ University of Twente, The Netherlands
}

\begin{abstract}
Knowledge graphs are a new form of knowledge representation. They are closely related to semantic networks and can be looked upon as in line with Schank's conceptual dependency theory and Sowa's conceptual graphs. The special feature of knowledge graphs is the use of a very restricted set of types of relations, that is considered to be the basic set of primitive relations. The theory of knowledge graphs is outlined in the first part of the paper. In the second part the possibilities of knowledge graphs for solving problems posed by Electronic (Study) Books will be discussed.
\end{abstract}

Keywords: Electronic book; Graphs; Knowledge; Knowledge representation; Modelling; Relation.

\section{Knowledge graphs}

Graphs $G(\mathcal{V}, \mathcal{E})$ consist of a set $\mathcal{V}$ of elements, called vertices, and a set $\mathcal{E}$ of unordered pairs of elements of $\mathcal{V}$, called edges. Both vertices and edges may carry labels, thus giving rise to a labelled graph. Directed graphs $G(\mathcal{V}, \mathcal{A})$ consist of a vertex set $\mathcal{V}$ and a set $\mathcal{A}$ of ordered pairs of $\mathcal{V}$, called arcs, i.e. $\mathcal{A}$ is a subset of the Cartesian product $\mathcal{L} \mathcal{V}$. The reader is referred to any text book on graph theory, for instance Harary (1972) or Bondy and Murty (1976) for terminology. We will consider a mixed form in which both edges and arcs occur. The graphs are considered to be finite, i.e. sets $\mathcal{V}, \mathcal{E}$ and $\mathcal{A}$ have finite cardinality.

The graph structure (Fig, 1) is a very convenient way of representing concepts, as vertices, and relationships between them, as edges or arcs. The labels of such a graph are the words with which the concepts and the relationships are described. The resulting labelled graph is often called a semantic network. Semantic networks have been introduced by Quillian (1968); however, the idea of representing knowledge in this way is so natural that it has been developed under different names by many others. It is the basis of Schank's conceptual dependency theory (1982) and Sowa's conceptual graphs (1984). The various problems related to using graphs for representational purposes are extensively treated in the latter reference. These problems concern very old discussion points about the nature of a

Correspondence: Cornelis Hoede, Department of Applied Mathematics, University of Twente, P.O. Box 217, 7500 AE Enschede, The Netherlands. 
concept, its extension (all entities deserving the name) and its intention (all properties), and the semantics of the representational structure. Both merit remarks here.

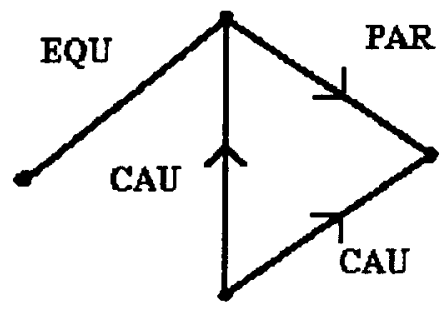

Fig. 1. Labelled mixed graph on four vertices with three arcs and one edge.

In the theory of knowledge graphs a concept is a labelled vertex. Any entity deserving the name, together with the properties that form the intention of the concept, is supposed to be representable in a graph. These are also represented by labelled vertices and their relation to the concept considered is expressed by the labelled edges or arcs. (The choice of the labels of these relationships will be discussed later.)

For the semantics we have the problem that there are competing schools of thought. On one side there is formal or logical semantics in which the semantics of a sentence, or its representation by a knowledge graph, is given by a model, describing the statement in the sentence, and a specification of the truth value of that model. The constituent parts of the sentence are modelled as propositions. The model corresponding to the sentence then has a truth value that can be calculated from the composition of the sentence in terms of its constituents. In this way the semantics of a sentence follows from the semantics of its parts. This is called the compositionality principle. On the other side there is cognitive semantics that stresses the fact that the bestowment of meaning to a sentence is done by the mind (person) that perceives the sentence. A typical representative of this school is Jackendoff (1983). The semantics of a knowledge graph has two components, the structural component, which is the graph itself, and a logical component or a cognitive component, depending on whether the truth assignment, to the graph interpreted as a proposition, is by agreement or by a single person.

\section{Specification of types of relationships}

A more precise description of knowledge graphs should be read with the foregoing analysis in mind. A concept may be analyzed by using a lexicon. In the end some primitive notion, like 'something', is met. One may also find oneself walking around in a cycle of synonyms. Considering everything to be something one introduces a vertex, without label, represented by a square, called token. All labelled vertices will be represented by a word in a rectangle. These vertices are called types. Next to tokens and types we have a restricted set of relationships, both symmetrical and asymmetrical. 
These relationships have types too. These are : EQU, PAR, ALI, ASS, CAU, ORD, DIS, SKO and FRM. To understand these types, to discuss their semantics, they must be seen as parts of the model as considered in cognitive semantics. The perceiver is considered to be able to decide on the truth assignment to a part of the knowledge graph. If this part is an edge or an arc its type label should be such that it may be considered to enable the person to determine whether indeed the suggested relationship is true. Also as a group we may decide in this matter, thus essentially making a model of the way we relate concepts. It will be clear that the choice of these types reflects our thinking. The types should therefore be very suggestive if they are to be acceptable as primitives. Their description, in normal words, is

- EQU: is equal to

- PAR: is part of

- ALI: is alike to (has things in common with)

- ASS: is associated with

- CAU: is causally related to

- ORD: is ordered with respect to

- DIS: is disparate from

- SKO: refers to the logician Skolem and may be described as 'is quantifiably related to'

- FRM: is in a part-frame relation to

The first four types may be interpreted as the four ways two sets (of attributes) may be related. There are, however, still problems here as, for example, the PAR-relationship type is still hotly debated, (see Iris et al., 1988). The element inclusion, the subset inclusion and the part-whole relationship may be seen as described by the PAR. Either the primitive PAR should be discernible in all three of them, or there are more primitive PARrelationship types than one.

The next two types, CAU and ORD, are disputable, especially the CAU. Hume - in the 18th Century - (Russell, 1961) - disputed the causal relation as a primitive. The ordering relation seems more primitive to him (and to the author). But even if CAU would turn out on further investigation to be composite, its great importance for the description of processes warrants inclusion in the list.

DIS enables one to express negation as was shown by van den Berg (1990). Van den Berg and Willems (1990) introduced the SKO type to describe 'all', 'each', 'every' and 'any' in terms of graphs.

Finally, a frame is a concept that has an inner structure that itself is described by a knowledge graph. The collection of attributes is a very simple example of an inner structure. Its graph consists of separate vertices. In this case, $a$ FRM $x$ expresses that the attribute $a$ is a frame part of the concept $x$. In this special case the PAR-relationship might also be used. For a more detailed discussion of knowledge graph theory, the reader is referred to James (1992). 


\section{Representing knowledge from books}

Before discussing the possibility of using the knowledge graph structure as an organizational principle for Electronic Books, the way that knowledge from texts is extracted and structured should be discussed briefly. In the Knowledge Integration and Structuring System (KISS), the following procedures are considered: text analysis, concept identification, link integration and construct analysis. We will describe what these procedures do.

Suppose some papers are available on a certain subject, then the process starts with the determination of concepts and the determination of relationships between them, e.g. by means of dictionaries of phrases that are considered to be indicators of certain types. This is one of the most difficult procedures in KISS. In principle one would need a complete dictionary for the extraction of all knowledge available in the papers. In practice only part of the contents of one paper is represented in the resulting author graph. Other texts on the subject then give other author graphs and these graphs may be pasted together by the process of concept identification. This procedure has the specific difficulty of the occurrence of synonyms. If concepts are to be identified, lists of synonyms must be available. However, in the system two concepts that have different names but similar contexts in the graphs are found by calculating similarity measures and may thereupon be identified by the user of the system. This yields a so-called basic graph, that can now be investigated on inferred relationships, by the process of link integration, and on relevant substructures, by the process of construct analysis.

In link integration a path algebra is used to infer the relationships that are not present in the basic graph but are implied by its structure. Construct analysis aims at discovering special substructures that may describe interesting, possibly new, concepts. This then, finally, leads to the so-called integrated graph. This graph represents the integrated and structured knowledge contained in the texts (Bakker, 1987 and de Vries, 1989). Two aspects that play an important role are consistency and robustness of the knowledge graph at any phase of its development (Smit, 1991). Consistency of a knowledge graph means that there are no substructures that lead to contradictions. An example is the occurrence of a cycle of PARrelationships, implying that a concept in this cycle is part of itself. Robustness of a knowledge graph expresses how sensitive the represented knowledge is to deletion of concepts and/or relationships. If, for example, a causal relationship is deleted but is inferred by the remaining graph, the graph is robust with respect to this deletion. If not, this relationship is a vital piece of information. The most important application of these ideas so far is the development of knowledge graphs that describe texts in medicine. The causal relationship plays a dominating role here. Such knowledge graphs can be used as an expert system for diagnosis, see for example the MEDES prototype (de Vries et al., 1988). 


\section{The representation of knowledge in Electronic Study Books}

The representation of knowledge in electronic books need not be in the form of a knowledge graph. It may be assumed that on consulting the knowledge in an electronic book, the user will like to read normal text on a computer screen, to see pictures or any other 'normal' piece of information. The whole account of knowledge graphs therefore seems to be besides the point, as in these graphs the vertices represent simple concepts, not complete texts. However, the organizational aspects of electronic books, the relations between pieces of information, are very similar, on a macroscopic level, to the organizational aspects of knowledge graphs, on a microscopic level. This also holds for representational systems like hypertext (Kommers, 1991).

We will now discuss the similarities between Knowledge Graphs and Electronic Study Book Platforms that De Diana (1991) describes as 'network environments for the use of Electronic Study Books'.

\section{Browsing}

De Diana mentions ease of browsing as a typical enrichment offered by an electronic book in comparison with a traditional (paper) book. The types of relationships that come into play here are the ORD and the structural relationship types EQU, PAR, ALI and ASS, next to FRM. One of the most primitive ways to describe the fact that two pieces of information are related is by using the ASS. This type simply expresses that one piece has something to do with the other piece of information without saying precisely what. A network of ASS-relationships between information units lets the user browse freely, without any help. Usually the information units have names and these cover detailed accounts. The relation between the unit and its detailed account is a typical example of a FRM-relationship. If 'vulcanism' is the name of the unit, a windowing process may display a detailed account of vulcanism.

A more intelligent electronic book will mimic the organization of the ordinary book in chapters, sections and paragraphs. These information units are hierarchically organized and the relation between them is of the type PAR. This organization can be represented by a graph in the form of a tree. The vertices denote the units and the arcs originate from the words that represent the book itself. The browser goes up and down in this tree and this may be called guided browsing. An interesting way of offering help in free browsing was developed by Kommers (op cit), who indicated for each unit the centrality of the concept in the network by its size on the screen, so that the user was aware of the fact that he was studying a concept of great or of little importance.

Let us now consider the situation in which, for a certain lecture, material is chosen from the material of a text book. Authors then indicate in which order a semester course may be composed from the material contained in the book, or how a two semester course may be composed. This aspect of the organization is well described by the ORD-relationship, the ordering being on the times at which the different units should be read. 
The EQU and the ALI relationship types come into play in browsing in footnotes that contain definitions of terms in the text or that refer to other texts with overlapping contents.

Thus the six types of relationship are able to describe the organization of the information units and it may be worthwhile to indicate on the screen the type of relationship the user is following in the browsing process.

\section{Information marking}

If certain units of information are marked we should make a distinction between two problems, one technical, the other to do with the study process itself.

The technical problem has two parts. The first is the most important from the point of view of Knowledge Graphs. If marking is done because extra information is added, e.g. that the unit is very important, then we have to deal with a special attribute of that unit. The PAR relationship expresses the attribute-unit relation and that is all. The second part of the problem is the way the marking is done; important units may be described in colour, capital letters or underlining which are issues of perception (cf. Kommers' solution for free browsing).

The second problem that De Diana (1991) mentions has to do with the study process itself. We can imagine that various forms of demarcation may be chosen at the beginning of the reading process. Suppose that one is mainly interested in the locations, sites and places that occur in the book; then only those will be marked which help the reader with his specific way of studying the book. Such marking would demand specific information behind every bit of information. To put it in words of Knowledge Graphs; every word would have to be described by a frame, in which attributes, like location, can be searched for in order to mark the specific words that are of interest.

More ambitious is marking depending on the use of the book so far. If a book is to be studied with accompanying exercises, the score of the reader may be used to mark those parts of the rest of the text that should be studied in detail, based on the performance of the reader up to that time. This involves really difficult educational problems.

\section{Annotation}

We already suggested that footnotes could be included by the producer using the structural types of relationships. There are no problems in allowing the reader to make annotations, except that he should know about the various types of relationships that may be used to link his remarks with the text in the book.

The possibility of multipersonal annotations brings us back to the heart of the Knowledge Graph project. If more and more pieces of information are linked to the existing bulk then this closely parallels the development of an integrated graph or at least a basic graph. The conflicts that these conditions 
may cause are central in the discussion of consistency of Knowledge Graphs. The DIS-relationship type plays a central role here. Activation of units of information means extension of the graph, while de-activation means deleting vertices and edges or arcs. In that case robustness of the whole book becomes an important issue. The demands mentioned by De Diana involve very difficult problems. However, it seems that, in principle, they can be met by Knowledge Graph theory, using the results of Smit (1990). Consider, for example, the requirement that the electronic book should allow selective activation and de-activation of annotation by the user. This seems to be a simple demand as it boils down to choosing a specific knowledge graph from a larger one. However, whether the knowledge represented is consistent and robust may depend on a few pieces of information, that may just happen to be part of the activated or de-activated material. This means that for every selection a complete analysis of the graph will have to be made taking account of consistency and robustness.

\section{Group-based learning, information sequencing and information distribution}

On these topics only a few remarks will be made. It is not entirely clear what is meant by group-based learning; it may mean that a group wants all its members to read the same book, while all members are making annotations. This means that a Knowledge Graph is perpetually changing shape. The KISS system that controls the integration and structuring process has been implemented and may be used for helping the group. So in this case the theory of Knowledge Graphs is helpful.

If, however, networked communication is not complete between all participants a problem of a different nature arises. In this case the vulnerability of the network is the central issue, and this may be investigated with ideas stemming from robustness considerations.

It has already been remarked that the ORD relationship can be used for information sequencing. The various problems concerning the prescription of ordering (sequencing) fall outside the scope of this paper.

The ultimate goal of Knowledge Graph theory is to describe the way in which all knowledge can be integrated into one huge graph. This goal clearly does not consider copyright issues. That these issues play an important role in information distribution is not denied, but the library of the next centuries will not consist of books but of integrated and structured units of information. The source of the information, which may be represented by an attribute 'name of author,' is the only thing that will remain to protect copyright. The subgraph determined by this attribute is the market share belonging to the author/publisher.

\section{Architecture}

This paper has discussed, almost exclusively, matters of the architecture of Electronic Book Study Platforms. We will therefore react to this part of De 
Diana's paper briefly by recalling some reactions, from the point of view of Knowledge Graphs, to his eight layer model of an Electronic Study Book.

- Layer 1. The basic materials are the vertices of the Knowledge Graph.

- Layer 2. Partitioning is done by PAR relationship types as well as by FRM.

- Layer 3. Marking also involves PAR in its special function of describing attribute 'importance'.

- Layer 4. Annotation involves EQU and ALI, and possibly, PAR and FRM.

- Layer 5. Group based activities involve integration and structuring which imply consistency and robustness. These aspects are described by, amongst others, DIS.

- Layer 6. Sequencing is taken care of by ORD.

- Layer 7. Control structure (see our account of browsing).

- Layer 8. Distribution (see our account of distribution).

\section{Adaptation}

There seems to be no essential difference between the processes of change involved in group-based learning and adaptation in other ways.

\section{Discussion}

As we hope to have shown the structure of Knowledge Graphs is essentially the same as that of Electronic Books. The only difference is the size of the units of information. In dealing with natural language also the CAU and the SKO-relationship came into play for the description of processes and quantification respectively. The author considers it a firm support for the paradigm of Knowledge Graphs that the other types of relationships all come forward in a natural way in the discussion of the organization of Electronic Books. Wherever and whenever Electronic Books are to be studied, the theory of Knowledge Graphs may offer considerable help.

\section{References}

Bakker, R.R. (1987) Knowledge graphs: Representation and structuring of scientific knowledge. PhD Thesis. University of Twente, Enschede.

Berg, H. van den (1990) Negation in knowledge graphs (Memorandum nr. 893). Faculty of Applied Mathematics, University of Twente, Enschede.

Berg, H. van den, \& M. Willems (1990) Quantification in knowledge graphs (Memorandum nr. 872). Faculty of Applied Mathematics, University of Twente, Enschede.

Bondy, J.A. \& U.S.R. Murty (1976) Graph theory with application. MacMillan Press, London.

De Diana, I.P.F. (1991) Electronic Study Book Platforms. Educational \& Training Technology International, 28, 4, 347-355.

de Vries, P.H. (1989) Representation of scientific texts in knowledge graphs. PhD Thesis. University of Groningen, Groningen. 
112 C. Hoede

de Vries Robbé, P.F., Beckers, W.P.A. \& Zanstra, P.E. (1988) MEDES, het prototype [MEDES, the prototype] (Rapport UHB/MIDS/EOO). Academisch Ziekenhuis Groningen, Groningen.

Harary, F. (1972) Graph theory. Addison-Wesley, Reading.

Iris, M.A., Litowitz, B.E. \& Evens M.W. (1988) Problems of the part-whole relation. In Relational models of the lexicon (ed. M. Walton Evens) pp. 261-288. Cambridge University Press, Cambridge.

Jackendoff, R. (1983) Semantics and cognition. MIT Press, Cambridge MA.

James, P. (1992) Knowledge graphs. In Linguistic instruments in knowledge engineering (eds. R.P. van de Riet \& R.A. Meersman). Elsevier Science Publishers, Amsterdam.

Kommers, P. (1991) Hypertext and the acquisition of knowledge. PhD Thesis. University of Twente, Enschede.

Quillian, R. (1968) Semantic memory. In Semantic Information Processing (ed. M. Minsky). MIT Press, Cambridge, MA.

Russell, B. (1961) History of western philosophy. Unwin University Books, London.

Schank, R.C. (1982) Dynamic Memory. Cambridge University Press, Cambridge.

Smit, H.J. (1991) Consistency and robustness of knowledge graphs. $\mathrm{PhD}$ Thesis. University of Twente, Enschede.

Sowa, J.F. (1984) Conceptual structures. Addison-Wesley, Reading. 